\title{
Discontinuous Galerkin method for multifluid Euler equations
}

\author{
Marc T. Henry de Frahan* \\ Eric Johnsen ${ }^{\dagger}$ \\ Mechanical Engineering Department, University of Michigan, Ann Arbor, MI 48109, USA
}

\begin{abstract}
Based on previous work extending the Discontinuous Galerkin method to multifluid flows, we analyze the performance of our numerical scheme. First, the advection of a contact discontinuity and a multifluid version of the Sod shock-tube problem are considered. We compare the oscillations generated by the non-conservative and conservative formulations of the ratio of specific heats equations, and demonstrate the non-oscillatory nature of the proposed numerical method. We compare the results for different proposed Riemann solvers. We verify the mass, momentum, and energy conservation properties of the scheme. Finally, we validate our numerical simulations against experimental results of the Richtmyer-Meshkov instability.
\end{abstract}

\section{Introduction}

$\mathrm{T}$ HE Discontinuous Galerkin (DG) method is a popular numerical method that has received much attention in recent years. This method for partial differential equations relies on a polynomial expansion to represent the discretized solution inside each cell of the domain. The DG method is a high-order accurate and compact-stencil scheme that combines the advantages of the finite element and finite volume (FV) methods. It is, therefore, a convenient method for parallel architectures and implementable on unstructured grids. The DG method has been succesfully adapted to solving time-dependent hyperbolic partial differential equations through the Runge-Kutta DG method. ${ }^{1-5}$ Since the flow solution is allowed to be discontinuous between cells, dissipation can be introduced where necessary, and, borrowing from the FV community, the traditional Riemann solvers can be used to compute numerical fluxes. Limiters have been developed to accurately capture shocks and discontinuities by damping the oscillations caused by high-order interpolation at the discontinuities. A total variation bounded limiter ${ }^{2}$ was designed to truncate the high-order coefficients at the discontinuities while keeping them when the flow is smooth. Subsequently, a hierarchical moment limiter reconstructing the Legendre coefficients from the highest to lowest moments was developed to avoid excessive dissipation. ${ }^{6}$ Various high-order limiters have followed and have been studied in recent years. ${ }^{7-10}$

The DG method has been applied successfully to single-fluid compressible flows. However, an accurate representation of multifluid flows is lacking in the DG framework. Some attention has been paid to the BaerNunziato system of equations for multiphase flows ${ }^{11,12}$ and the compressible Navier-Stokes equations. ${ }^{13}$ When solving the conservative form of transport equations for the ratio of specific heats, spurious pressure oscillations develop at the interface between the different fluids in FV methods. ${ }^{14}$ These oscillations can be avoided by solving a non-conservative form of the evolution equation for the ratio of specific heats. In the FV framework, many approaches to solve this problem have been proposed ${ }^{15,16}$ and extended to high-order accuracy. ${ }^{17-19}$

This paper is organized as follows. In Section II, we summarize the numerical tools necessary to accurately solve the multifluid Euler equations in the DG framework. In Section III, using the advection of a contact discontinuity and a multifluid version of the Sod shock tube problem, we compare different formulations for

${ }^{*} \mathrm{PhD}$ Candidate, University of Michigan, 1231 Beal Ave. 2043 Walter E. Lay Automotive Laboratory, Ann Arbor, MI 48109-2133

${ }^{\dagger}$ Assistant Professor, University of Michigan, 1231 Beal Ave. 2043 Walter E. Lay Automotive Laboratory, Ann Arbor, MI 48109-2133 
the ratio of specific heats and for different Riemann solvers, and validate the conservation properties of the numerical methods. Finally, we present two-dimensional simulation of the Richtmyer-Meshkov instability and compare the growth to experimental data.

\section{Numerical Analysis}

In this paper, we consider the Euler equations for perfect gases, written here in one dimension for simplicity:

$$
\frac{\partial}{\partial t}\left[\begin{array}{c}
\rho \\
\rho u \\
E
\end{array}\right]+\frac{\partial}{\partial x}\left[\begin{array}{c}
\rho u \\
\rho u^{2}+p \\
u(E+p)
\end{array}\right]=0
$$

where $\rho$ is the density, $u$ is the velocity, $E=p /(\gamma-1)+\rho u^{2} / 2$ is the total energy, $p$ is the pressure, and $\gamma$ is the potentially varying specific heats ratio. Here, diffusion is neglected. When considering multiple fluids, an additional (transport) equation must be solved for the fluid composition. To avoid the development of spurious pressure oscillations at an initially constant pressure interface between two perfect gases in the DG framework, we have shown that a non-conservative advection equation for $\gamma$,

$$
\frac{\partial}{\partial t}\left(\frac{1}{\gamma-1}\right)+u \frac{\partial}{\partial x}\left(\frac{1}{\gamma-1}\right)=0
$$

must be solved. ${ }^{20}$ This is analogous to similar findings for the FV framework. ${ }^{14}$

\section{II.A. Discontinuous Galerkin Methods for Non-Conservative Laws}

Theoretical investigations and numerical solutions for weak solutions of equations containing non-conservative products have been presented in the FV context. ${ }^{21-25}$ We follow a DG formulation for non-conservative laws. $^{26,27}$ For the one-dimensional hyperbolic partial differential equation,

$$
\frac{\partial W}{\partial t}+\frac{\partial F}{\partial x}+G \frac{\partial W}{\partial x}=0
$$

the DG weak formulation is obtained by integrating inside each cell $\Omega_{j}=\left[x_{j-1 / 2}, x_{j+1 / 2}\right]$, and taking the inner

product with a test function, $\varphi_{i}$,

$$
\int_{\Omega} \varphi_{i} \frac{\partial W}{\partial t} \mathrm{~d} x=\int_{\Omega} \frac{\mathrm{d} \varphi_{i}}{\mathrm{~d} x} F \mathrm{~d} x-\int_{\Omega} \varphi_{i} G \frac{\partial W}{\partial x} \mathrm{~d} x-\left[\llbracket \varphi_{i} \rrbracket \hat{F}-\left\{\left\{\varphi_{i}\right\}\right\} \frac{\partial W}{\partial x}\right]_{x_{j-1 / 2}}^{x_{j+1 / 2}},
$$

where $\hat{F}$ is a numerical flux at the cell edges, $\llbracket \alpha \rrbracket=\alpha_{L}-\alpha_{R},\left\{\{\alpha\}=\frac{1}{2}\left(\alpha_{L}+\alpha_{R}\right)\right.$, and $L$ and $R$ are the left and right states at the cell edges. The DalMaso-LeFloch-Murat theory ${ }^{22}$ determines

$$
\left[G \frac{\partial W}{\partial x}\right]_{x_{j+1 / 2}}=\int_{0}^{1} G\left(\phi\left(\tau ; W^{L}, W^{R}\right)\right) \frac{\partial \phi}{\partial \tau}\left(\tau ; W^{L}, W^{R}\right) \mathrm{d} \tau
$$

where $\phi$ is a path in phase space relating the left state to the right state.

Numerical fluxes for conservation laws are determined with the traditional Riemann solvers. For the non-conservative products, an $\mathrm{HLL}^{28}$-like flux was developed ${ }^{26}$ and applied to Eq. (4):

$$
\hat{F}= \begin{cases}F^{L}-\frac{1}{2} \int_{0}^{1} G\left(\phi\left(\tau ; W^{L}, W^{R}\right)\right) \frac{\partial \phi}{\partial \tau}\left(\tau ; W^{L}, W^{R}\right) \mathrm{d} \tau & \text { if } S_{L}>0, \\ \left\{\{F\}+\frac{1}{2}\left(S_{R} W^{*}+S_{L} W^{*}-S_{L} W^{L}-S_{R} W^{R}\right)\right. & \text { if } S_{L}<0<S_{R}, \\ F^{R}+\frac{1}{2} \int_{0}^{1} G\left(\phi\left(\tau ; W^{L}, W^{R}\right)\right) \frac{\partial \phi}{\partial \tau}\left(\tau ; W^{L}, W^{R}\right) \mathrm{d} \tau & \text { if } S_{R}<0,\end{cases}
$$

where $S_{L}$ and $S_{R}$ are the velocities of the fastest left and right moving waves in the system, and

$$
W^{h l l}=\frac{S_{R} W^{R}-S_{L} W^{L}+F^{L}-F^{R}}{S_{R}-S_{L}}-\frac{1}{S_{R}-S_{L}} \int_{0}^{1} G\left(\phi\left(\tau ; W^{L}, W^{R}\right)\right) \frac{\partial \phi}{\partial \tau}\left(\tau ; W^{L}, W^{R}\right) \mathrm{d} \tau .
$$


We have extended this idea ${ }^{20}$ and proposed Lax-Friedrichs ${ }^{29}$ and Rusanov ${ }^{30}$ solvers for Eq. (4),

$$
\hat{F}=\left\{\{F\}-\frac{S}{2}\left(W^{R}-W^{L}\right),\right.
$$

where $S$ is the velocity of the fastest local wave in the system for Rusanov, or the fastest global wave in the domain for Lax-Friedrichs. We also proposed ${ }^{20}$ a Roe ${ }^{31}$ solver for Eq. (4), $\hat{F}=\left\{\{F\}-\frac{1}{2} \sum_{i} \hat{\alpha}_{i}\left|\hat{\lambda}_{i}\right| \hat{r}_{i}\right.$, where $\hat{r}_{i}$ are the right eigenvectors of the linearized Roe matrix $A_{\phi}, \hat{\lambda}_{i}$ are the corresponding eigenvalues, and $\hat{\alpha}_{i}$ are the wave strengths. The left and right eigenvectors, the corresponding eigenvalues, and the strengths across the discontinuity have been established for the multifluid Euler equations. ${ }^{32}$

For the paths connecting the left and right states, we choose a linear path for the HLL, Rusanov, and Lax-Friedrichs solvers,

$$
\phi\left(\tau ; W^{L}, W^{R}\right)=\left(W^{R}-W^{R}\right) \tau+W^{L} .
$$

For the Roe solver, we do not explicitly define a path but assume it exists. ${ }^{20}$

Problems with shocks and discontinuities require a solution-limiting procedure to avoid numerical oscillations caused by interpolating across the discontinuity. In this work, we use a limiter based on the idea of hierarchical reconstruction (HR) ${ }^{9,}{ }^{10}$ In this procedure, the coefficients of the cell polynomial are limited from the highest to the lowest degree coefficient. The coefficients in a cell can be compared to the ones in the neighboring cells with the MUSCL ${ }^{33}$ or WENO ${ }^{34}$ approach for example. Limiting in this way ensures that the HR procedure is conservative and maintains high-order accuracy. Traditionally, to dissipate the oscillations at the discontinuities, HR is applied to the conserved variables $(\rho, \rho u, E)$. This strategy introduces spurious pressure oscillations in flows with a varying ratio of specific heats. While limiting the primitive variables could avoid the development of pressure oscillations, ${ }^{17}$ this would be lead to the loss of the conservation property of the scheme. ${ }^{20}$ Therefore, we have proposed a modified HR procedure that ensures that the scheme is both conservative and non-oscillatory. ${ }^{20}$ The idea is to limit $\rho, \rho u, p$, and $1 /(\gamma-1)$ using traditional hierarchical reconstruction. The energy coefficients are then reconstructed with these fields according to a formula derived to ensure that both the energy is conserved and the scheme is non-oscillatory. The additional computational cost of this procedure is small compared to the overal HR procedure. ${ }^{20}$

\section{Numerical Tests}

In this section, we consider the advection of a contact discontinuity and the multifluid Sod problem to compare the different $\gamma$ formulations and different Riemann solvers, and to establish the conservation property of the numerical scheme. We present initial investigations of the temperature oscillations that can develop at the fluid interface. We also validate our approach against experimental results of the RichtmyerMeshkov instability. For these problems, we use the modified HR procedure when limiting is required and the standard fourth order Runge-Kutta method with $\mathrm{CFL}<10^{-1}$ to avoid time-stepping errors.

\section{III.A. Contact Discontinuity: Oscillations and Conservation}

In this section, we consider the advection of a contact discontinuity in a single fluid. We solve the Euler equations in the periodic domain $x \in[-1,1]$, along with either the non-conservative form, Eq. (2), or the following conservative equation:

$$
\frac{\partial}{\partial t}\left(\frac{\rho}{\gamma-1}\right)+\frac{\partial}{\partial x}\left(\frac{\rho u}{\gamma-1}\right)=0
$$

The initial conditions are given by

$$
(\rho, u, p, \gamma)^{L}=(1,1,1,1.4), \quad(\rho, u, p, \gamma)^{R}=(0.125,1,1,1.4) .
$$

The $L_{\infty}$ cell-average error of velocity and pressure as a function of spatial resolution is shown in Figure 2. It can be observed that the non-conservative formulation does not present spurious pressure oscillations in velocity and pressure, Figure 1. These are clearly present when solving the conservative formulation for $\gamma$. This might be due to the inconsistent treatment of density when limiting the conservative formulation for $\gamma$. Similar results have been described in the FV and finite difference frameworks. ${ }^{35}$ Figure 3 shows the relative error in total mass, momentum, and energy as a function of time in the system. These three quantities are conserved by the numerical scheme. 


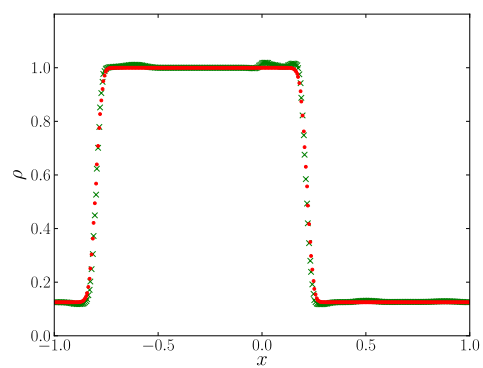

(a) Density

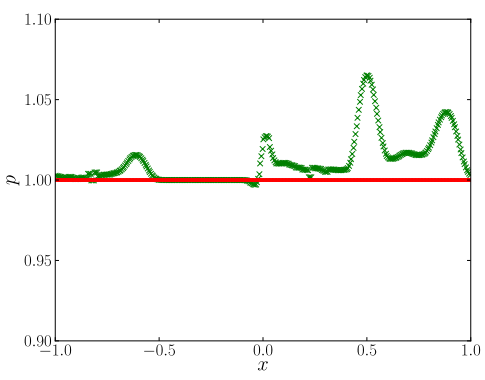

(c) Pressure

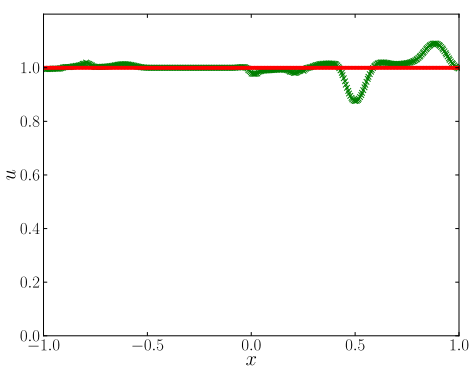

(b) Velocity

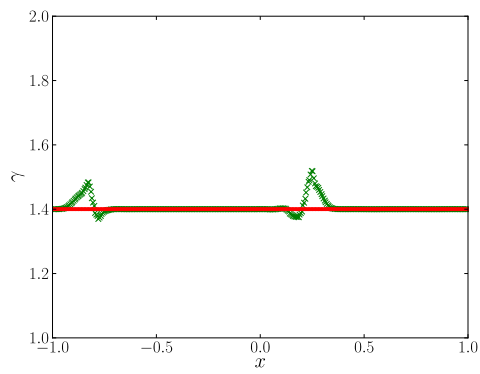

(d) Ratio of specific heats

Figure 1: Profiles at $t=0.2$ for the advection of a contact discontinuity in a single fluid $(p=2, \Delta x=1 / 64$, Roe flux). Red dots: non-conservative; green crosses: conservative.

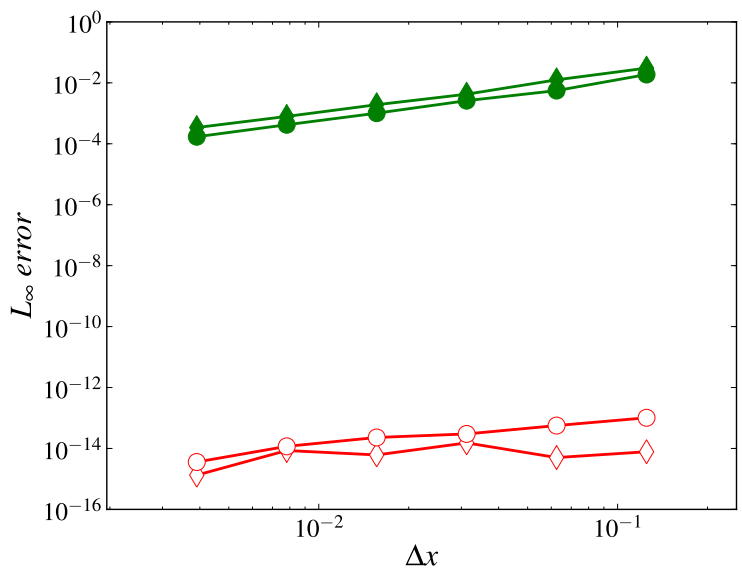

Figure 2: $L_{\infty}$ cell-average error of velocity (diamonds) and pressure (circles) vs. $\Delta x$ for the advection of a contact discontinuity ( $p=2$, Roe solver). Empty red: non-conservative; filled green: conservative. 


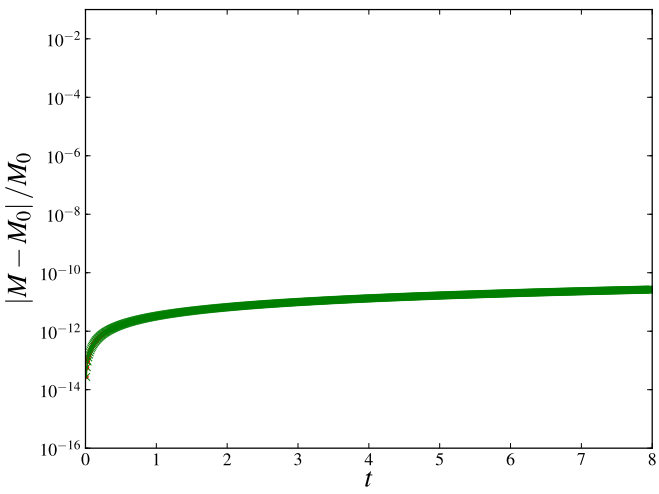

(a) Total mass

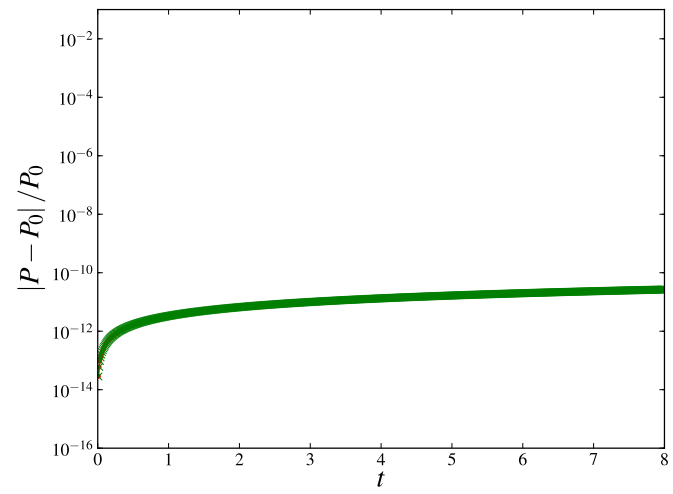

(b) Total momentum

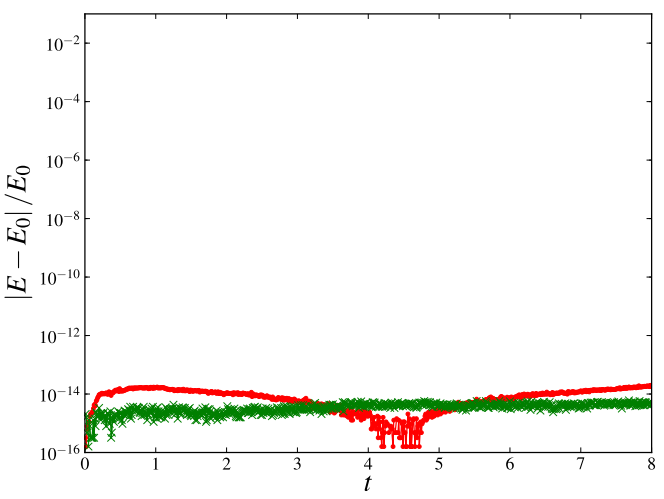

(c) Total energy

Figure 3: Relative error in total mass, momentum, and energy as a function of time $(p=2, \Delta x=1 / 64$, Roe solver). Red dots: non-conservative; green crosses: conservative. 


\section{III.B. Two-fluid Sod Shock Tube: Oscillations and Conservation}

This test problem is a multifluid version of the Sod shock tube problem. ${ }^{16}$ We solve the multifluid Euler equations in the open domain $x \in[-1,1]$ and compare the two formulations for $\gamma$. The initial conditions are

$$
(\rho, u, p, \gamma)^{L}=(1,0,1,1.4), \quad(\rho, u, p, \gamma)^{R}=(0.125,0,0.1,1.6) .
$$

Figure 4 shows the density, velocity, pressure and $\gamma$ profiles at $t=0.2$ for $p=2$. Oscillations in velocity and pressure, and overshoots in $\gamma$, are clearly observed with the conservative formulation. These are absent in the non-conservative formulation. The overshoots and undershoots in $\gamma$ in the conservative formulation are most likely due to the initial velocity spike at the start of the problem. These are then dissipated by the Riemann solvers to the point where they are barely visible for the Rusanov solver, yet still very much present for the other two solvers (Figures $4 \mathrm{j}, 4 \mathrm{k}$, and $4 \mathrm{l}$ ). Although the HLL-like flux presents a slightly more oscillatory solution, Figure 4h, the Riemann solvers predict a qualitatively similar solution. Additionally, the dissipative nature of the Riemann solvers is evident in Figures 4a, 4b, and 4c, from the more dissipative Rusanov flux to the least dissipative Roe flux.

The relative error in total mass, momentum, and energy for the multifluid Sod problem in a periodic domain over eight periods is shown in Figure 5 for $p=2$. Both the conservative and non-conservative formulations conserve the total mass, momentum, and energy in the system. 


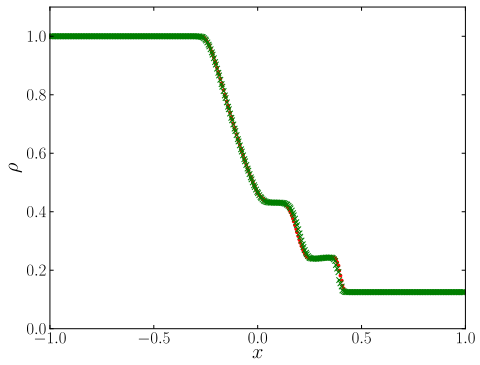

(a) Density

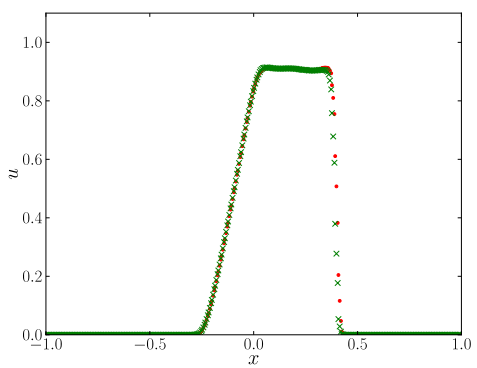

(d) Velocity

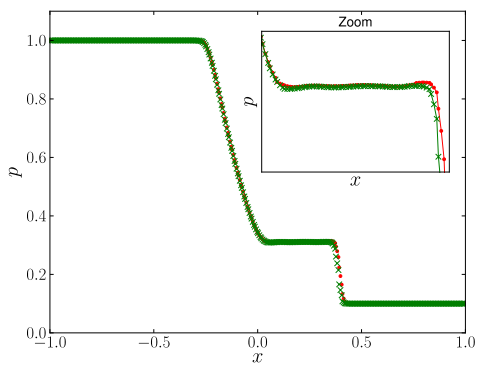

(g) Pressure

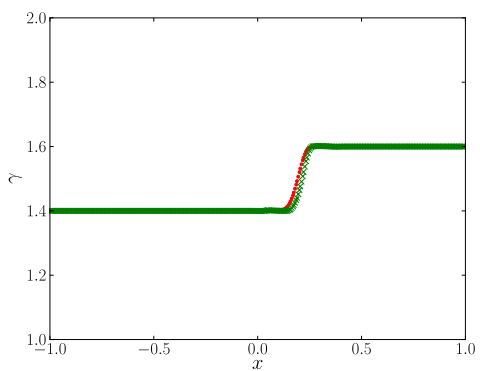

(j) Ratio of specific heats

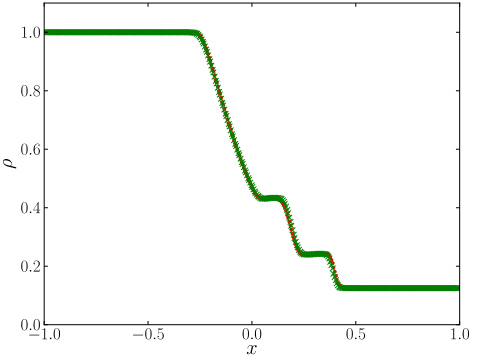

(b) Density

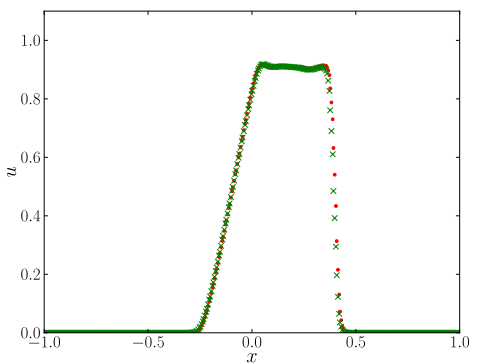

(e) Velocity

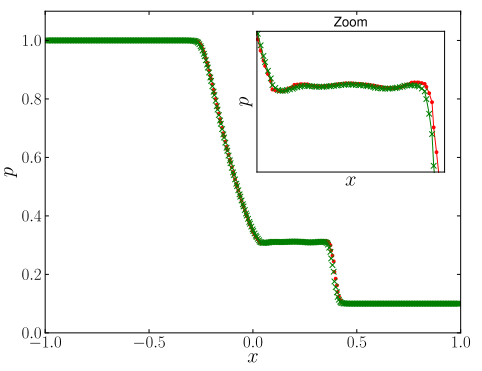

(h) Pressure

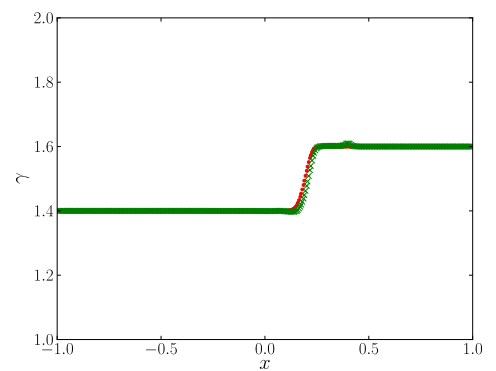

(k) Ratio of specific heats

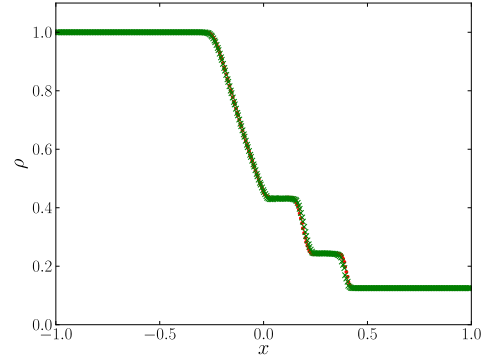

(c) Density

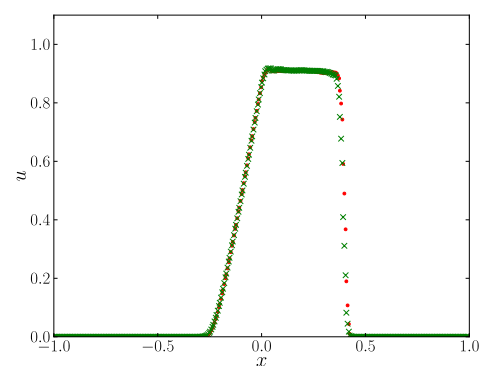

(f) Velocity

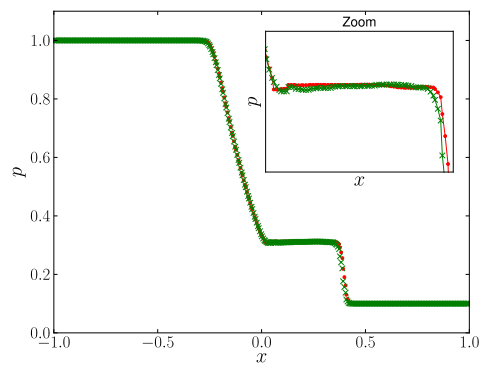

(i) Pressure

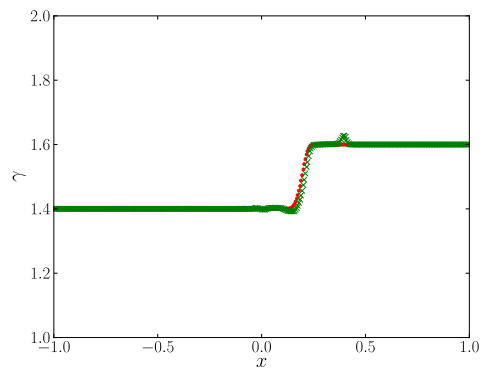

(l) Ratio of specific heats

Figure 4: Profiles at $t=0.2$ for the multifluid Sod problem $(p=2, \Delta x=1 / 64)$. Red dots: non-conservative; green crosses: conservative. Left: Rusanov flux; center: HLL-like flux; right: Roe flux. 


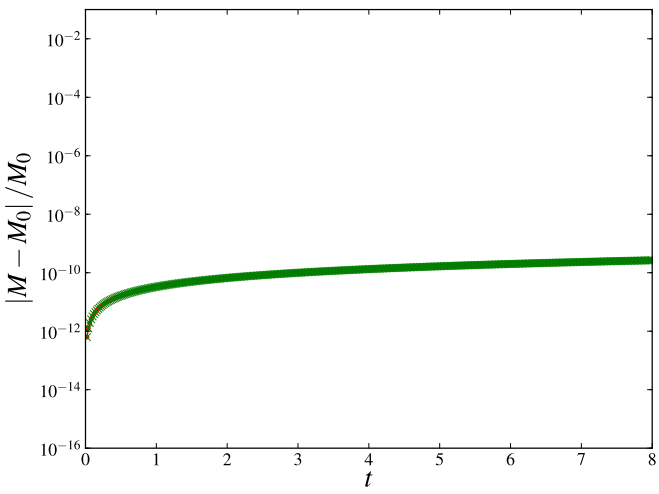

(a) Total mass

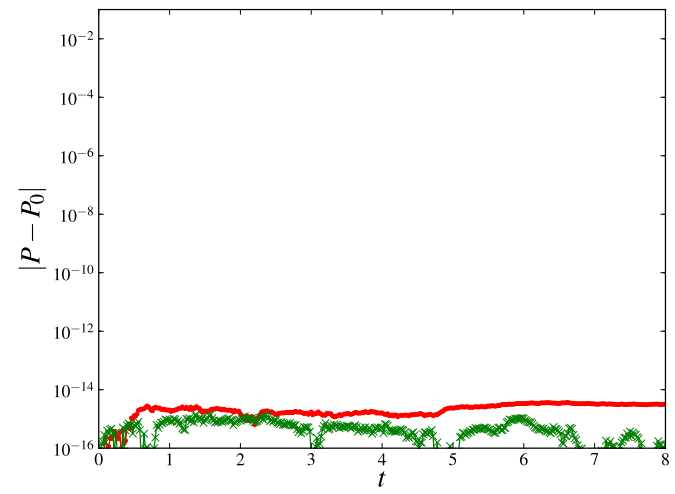

(b) Total momentum

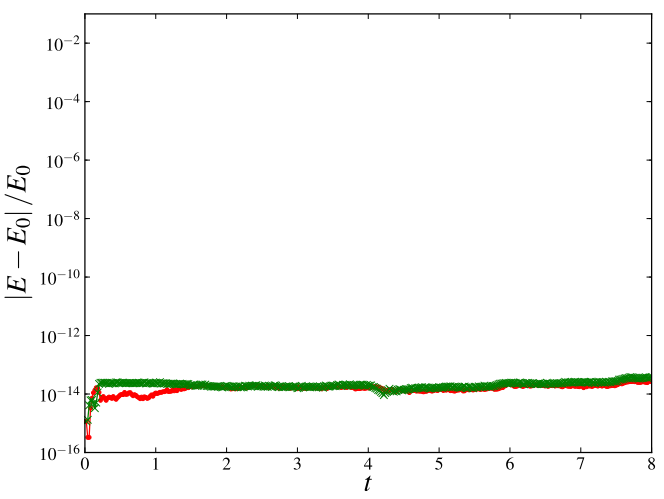

(c) Total energy

Figure 5: Relative error in total mass, momentum, and energy as a function of time $(p=2, \Delta x=1 / 64$, Roe solver). Red dots: non-conservative; green crosses: conservative. 


\section{III.C. Two-dimensional Validations: Richtmyer-Meshkov Instability}

In this section, we validate our numerical methods with experiments. We compare our simulations to single-mode Richtmyer-Meshkov instability (RMI) experiments of a perturbed air-SF 6 interface. ${ }^{36}$ As in the experiment, the simulation perturbation wavelength is $5.933 \mathrm{~cm}$ and the initial perturbation amplitude is $0.183 \mathrm{~cm}$. The density of the air is $\rho_{\text {air }}=1.351 \mathrm{~kg} / \mathrm{m}^{3}$, its specific heats ratio is $\gamma_{\text {air }}=1.276$, and its molecular mass is $M_{\text {air }}=34.76 \mathrm{~kg} / \mathrm{kmol}$. The $\mathrm{SF}_{6}$ density is $\rho_{S F_{6}}=5.494 \mathrm{~kg} / \mathrm{m}^{3}$, its specific heats ratio $\gamma_{S F_{6}}=1.093$, and its molecular mass is $M_{S F_{6}}=146.05 \mathrm{~kg} / \mathrm{kmol}$. We emphasize that unlike some prior work $^{37}$ we are considering variable specific heats. We use our proposed method in two-dimensions with traditional HR.

A Mach 1.21 shock, initialized in air, Figure 6a, impinges upon an air-SF 6 interface and initiates the RMI growth by depositing baroclinic vorticity at the interface, Figure 6b. The RMI grows linearly at first and the transmitted shock leaves the domain, Figure 6c. The non-linear growth and appearances of secondary instabilities can be observed in Figures $6 \mathrm{~d}, 6 \mathrm{e}$, and $6 \mathrm{f}$. To validate our code, we compare the amplitude of the instability to experimental data, Figure 7. We observe that there is good agreement between our results and the experiments.

\section{Conclusions}

We have shown that, by solving the non-conservative formulation for $1 / \gamma-1$, we can avoid spurious pressure oscillations that arise at the interface separating different fluids. The three Riemann solvers previously proposed predict qualitatively similar solutions. In addition to being non-oscillatory, the scheme is conservative. Further investigation of the temperature profiles reveal oscillations that will be fixed in future work. On the basis of this study we will perform multi-dimensional and multiphysics simulations with the DG method.

\section{Acknowledgements}

The authors gratefully acknowledge discussions with Sreenivas Varadan and Rui Fang. This research was supported by the DOE NNSA/ASC under the predictive Science Academic Alliance Program by Grant No. DEFC52-08NA28616.

\section{References}

\footnotetext{
${ }^{1}$ Cockburn, B., Lin, G., and Shu, C.-W., "TVB Runge-Kutta local projection discontinuous Galerkin finite element method for conservation laws III: One-dimensional systems," J. Comput. Phys., Vol. 84, No. 1, 1989, pp. 90-113.

${ }^{2}$ Cockburn, B. and Shu, C.-W., "TVB Runge-Kutta local projection discontinuous Galerkin finite element method for conservation laws II: General framework," Math. Comput., Vol. 52, No. 186, 1989, pp. 411-435.

${ }^{3}$ Cockburn, B., Hou, and Shu, C.-W., "The Runge-Kutta local projection discontinuous Galerkin finite element method for conservation laws IV: The multidimensional case," Math. Comput., Vol. 54, No. 190, 1990, pp. 545-581.

${ }^{4}$ Cockburn, B. and Shu, C.-W., "The local discontinuous Galerkin method for time-dependent convection-diffusion systems," SIAM J. Numer. Anal., Vol. 35, No. 6, 1997, pp. 2440-2463.

${ }^{5}$ Cockburn, B. and Shu, C.-W., "The Runge-Kutta discontinuous Galerkin method for conservation laws V: Multidimensional systems," J. Comput. Phys., Vol. 141, No. 2, 1997, pp. 199-224.

${ }^{6}$ Biswas, R., Devine, K. D., and Flaherty, J. E., "Parallel, adaptive finite element methods for conservation laws," Appl. Numer. Math., Vol. 14, No. 1-3, April 1994, pp. 255-283.

${ }^{7}$ Krivodonova, L., "Limiters for high-order discontinuous Galerkin methods," J. Comput. Phys., Vol. 226, No. 1, Sept. 2007, pp. 879-896.

${ }^{8}$ Kuzmin, D., "A vertex-based hierarchical slope limiter for p-adaptive discontinuous Galerkin methods," J. Comput. Appl. Math., Vol. 233, No. 12, April 2010, pp. 3077-3085.

${ }^{9}$ Liu, Y., Shu, C.-W., Tadmor, E., and Zhang, M., "Central discontinuous Galerkin methods on overlapping cells with a nonoscillatory hierarchical reconstruction," SIAM J. Numer. Anal., Vol. 45, No. 6, 2007, pp. 2442.

${ }^{10} \mathrm{Xu}, \mathrm{Z}$., Liu, Y., and Shu, C.-W., "Hierarchical reconstruction for discontinuous Galerkin methods on unstructured grids with a WENO-type linear reconstruction and partial neighboring cells," J. Comput. Phys., Vol. 228, No. 6, April 2009, pp. 2194-2212.

${ }^{11}$ Tokareva, S. and Toro, E., "HLLC-type Riemann solver for the BaerNunziato equations of compressible two-phase flow," J. Comput. Phys., Vol. 229, No. 10, May 2010, pp. 3573-3604.

${ }^{12}$ Franquet, E. and Perrier, V., "RungeKutta discontinuous Galerkin method for interface flows with a maximum preserving limiter," Comput. Fluids, Vol. 65, July 2012, pp. 2-7.
} 


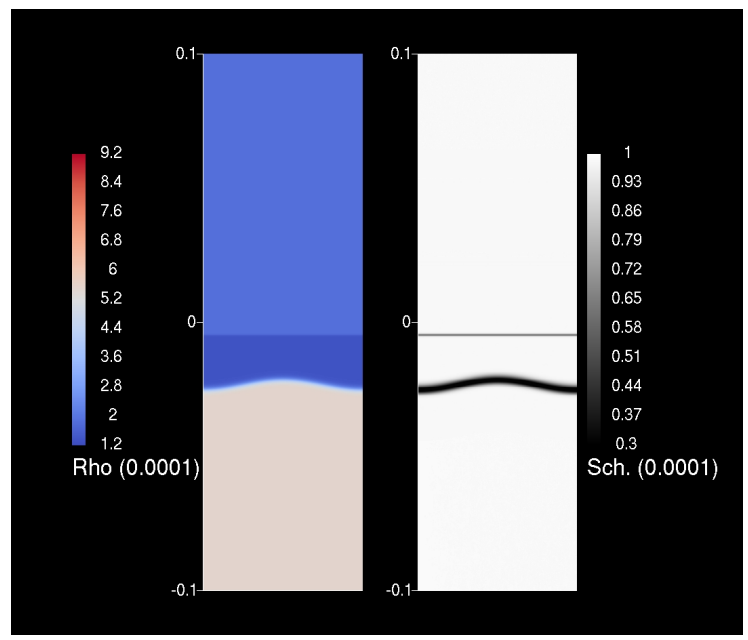

(a) Initial condition

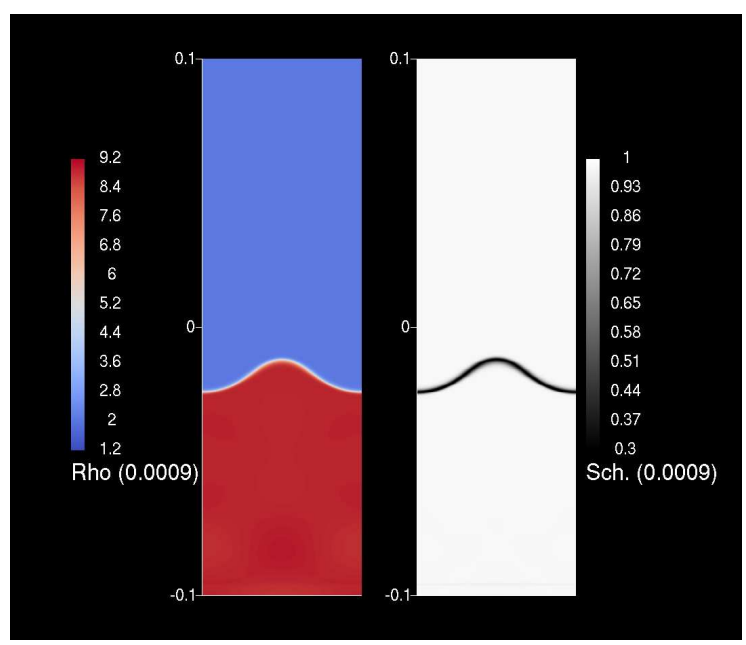

(c) $t=9 \mathrm{~ms}$

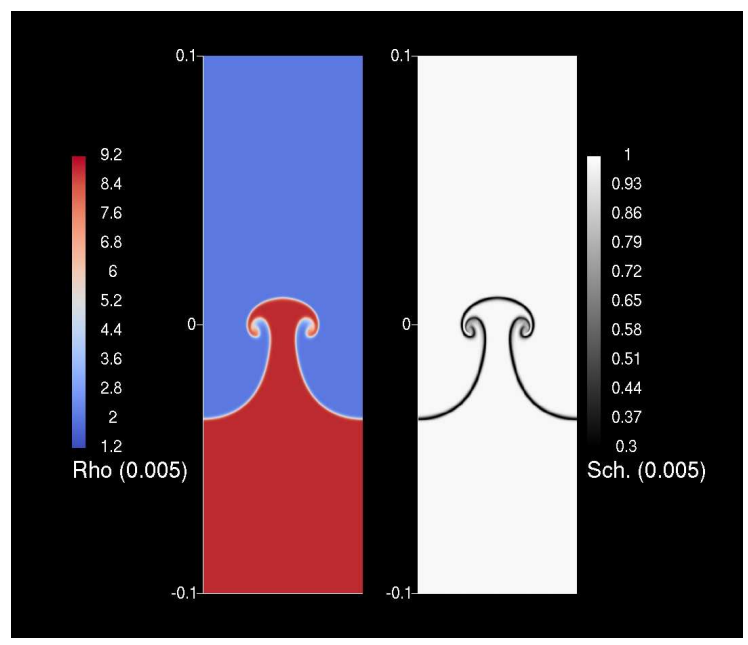

(e) $t=50 \mathrm{~ms}$

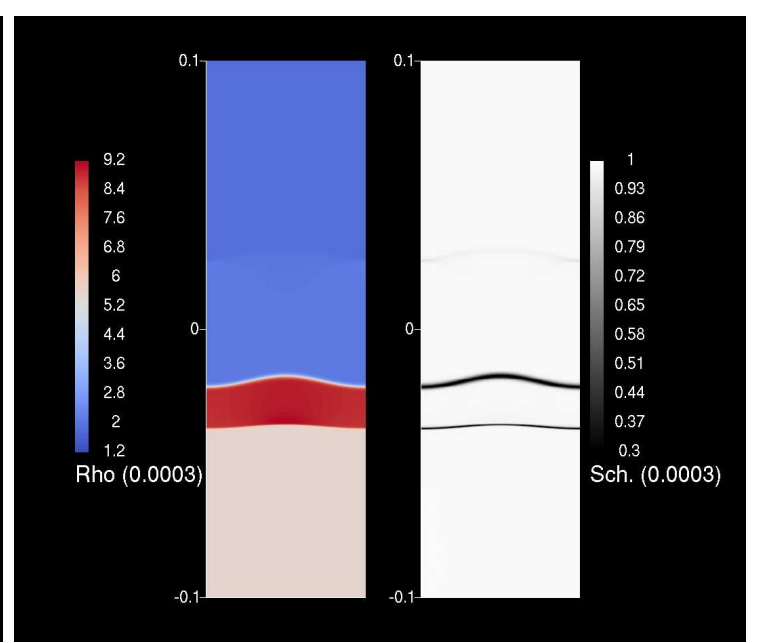

(b) $t=3 \mathrm{~ms}$

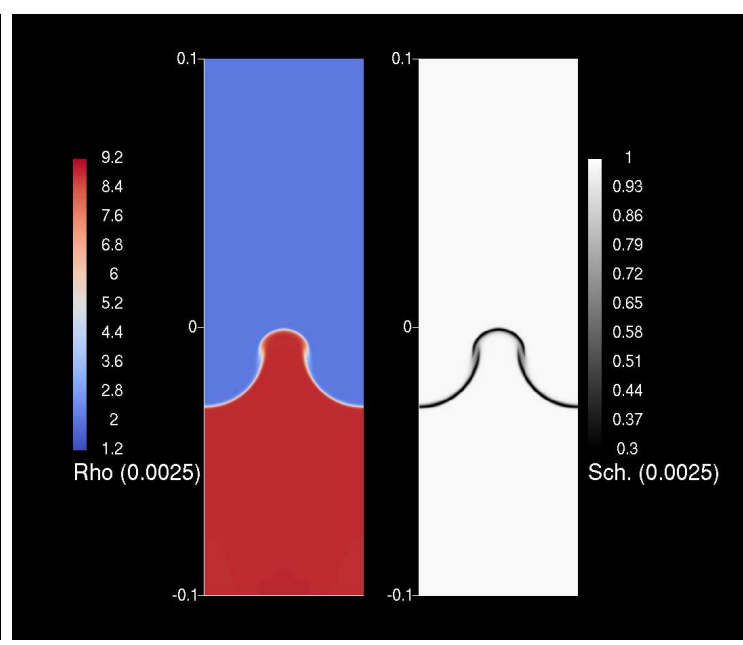

(d) $t=25 \mathrm{~ms}$

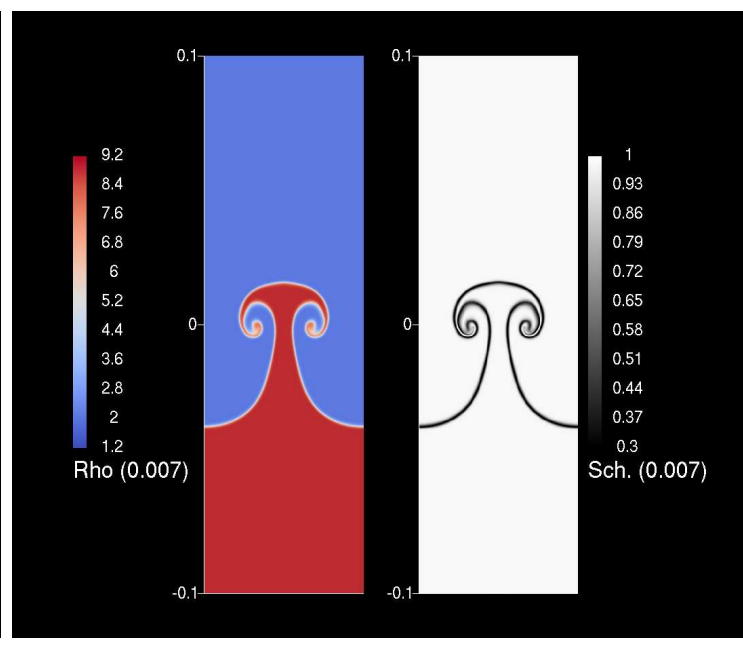

(f) $t=70 \mathrm{~ms}$

Figure 6: Density and simulated density Schlieren fields. 


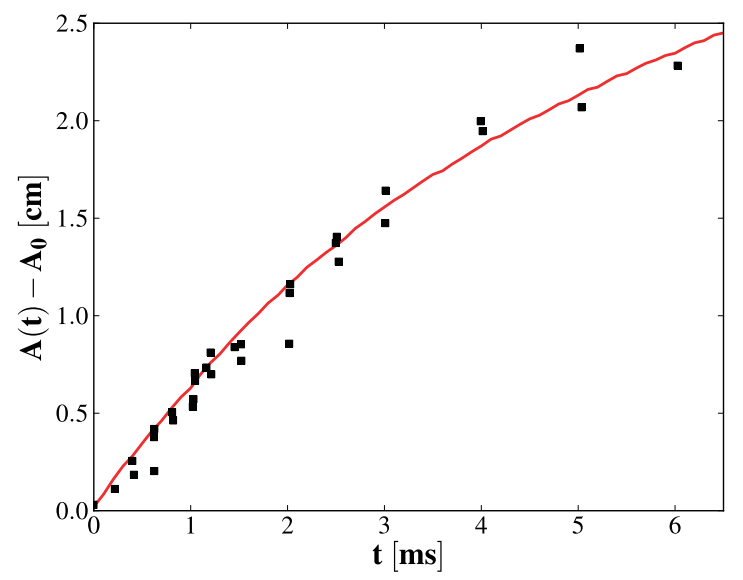

Figure 7: Instability growth as a function of time. Black squares: experimental data. ${ }^{36}$ Solid red line: simulation results.

\footnotetext{
${ }^{13}$ Michoski, C., Evans, J., Schmitz, P., and Vasseur, A., "A discontinuous Galerkin method for viscous compressible multifluids," J. Comput. Phys., Vol. 229, No. 6, March 2010, pp. 2249-2266.

${ }^{14}$ Abgrall, R., "How to prevent pressure oscillations in multicomponent flow calculations: A quasi conservative approach," J. Comput. Phys., Vol. 125, No. 1, April 1996, pp. 150-160.

${ }^{15}$ Saurel, R. and Abgrall, R., "A simple method for compressible multifluid flows," SIAM J. Sci. Comput., Vol. 21, No. 3, 1999, pp. 1115-1145.

${ }^{16}$ Abgrall, R. and Karni, S., "Computations of compressible multifluids," J. Comput. Phys., Vol. 169, No. 2, May 2001, pp. 594-623.

${ }^{17}$ Johnsen, E. and Colonius, T., "Implementation of WENO schemes in compressible multicomponent flow problems," $J$. Comput. Phys., Vol. 219, No. 2, Dec. 2006, pp. 715-732.

${ }^{18}$ Xiong, T., Shu, C.-W., and Zhang, M., "WENO Scheme with Subcell Resolution for Computing Nonconservative Euler Equations with Applications to One-Dimensional Compressible Two-Medium Flows," J. Sci. Comput., Vol. 53, No. 1, Feb. 2012, pp. 222-247.

${ }^{19}$ Movahed, P. and Johnsen, E., "A solution-adaptive method for efficient compressible multifluid simulations, with application to the RichtmyerMeshkov instability," J. Comput. Phys., Vol. 239, April 2013, pp. 166-186.

${ }^{20}$ Henry de Frahan, M. T. and Johnsen, E., "Interface capturing for multifluid simulations with shocks using Discontinuous Galerkin approaches," 2012, submitted.

${ }^{21}$ LeFloch, P. G., "Shock waves for nonlinear hyperbolic systems in nonconservative form," Institute for Math. and its Appl., Minneapolis, preprint, Vol. 593, 1989, pp. 1989.

${ }^{22}$ Dal Maso, G., LeFloch, P. G., and Murat, F., "Definition and weak stability of nonconservative products," J. Math. Pures Appl., Vol. 74, No. 6, 1995, pp. 483-548.

${ }^{23}$ Parés, C., "Numerical methods for nonconservative hyperbolic systems: A theoretical framework," SIAM J. Numer. Anal., Vol. 44, No. 1, 2006, pp. 300.

${ }^{24}$ Castro, M. J., LeFloch, P. G., Muñoz Ruiz, M. L., and Parés, C., "Why many theories of shock waves are necessary: Convergence error in formally path-consistent schemes," J. Comput. Phys., Vol. 227, No. 17, Sept. 2008, pp. 8107-8129.

${ }^{25}$ Canestrelli, A., Siviglia, A., Dumbser, M., and Toro, E. F., "Well-balanced high-order centred schemes for nonconservative hyperbolic systems. Applications to shallow water equations with fixed and mobile bed," Adv. Water Resour., Vol. 32, No. 6, June 2009, pp. 834-844.

${ }^{26}$ Rhebergen, S., Bokhove, O., and van der Vegt, J., "Discontinuous Galerkin finite element methods for hyperbolic nonconservative partial differential equations," J. Comput. Phys., Vol. 227, No. 3, Jan. 2008, pp. 1887-1922.

${ }^{27}$ Sollie, W., Bokhove, O., and van der Vegt, J., "Spacetime discontinuous Galerkin finite element method for two-fluid flows," J. Comput. Phys., Vol. 230, No. 3, Feb. 2011, pp. 789-817.

${ }^{28}$ Harten, A., Lax, P. D., and van Leer, B., "On upstream differencing and Godunov-type schemes for hyperbolic conservation laws," SIAM review, Vol. 25, No. 1, 1983, pp. 35-61.

${ }^{29}$ Lax, P. D., "Weak solutions of nonlinear hyperbolic equations and their numerical computation," Commun. Pure Appl. Math., Vol. 7, No. 1, Feb. 1954, pp. 159-193.

${ }^{30}$ Rusanov, V., "The calculation of the interaction of non-stationary shock waves and obstacles," Comput. Math. Math. Phys., Vol. 1, No. 2, 1962, pp. 304-320.

${ }^{31}$ Roe, P. L., "Approximate Riemann solvers, parameter vectors, and difference schemes," J. Comput. Phys., Vol. 43, No. 2, Oct. 1981 , pp. $357-372$.

${ }^{32}$ Shyue, K.-M., "An efficient shock-capturing algorithm for compressible multicomponent problems," J. Comput. Phys., Vol. 142, No. 1, May 1998, pp. 208-242.

${ }^{33}$ van Leer, B., "Towards the ultimate conservative difference scheme. V. A second-order sequel to Godunov's method," J. Comput. Phys., Vol. 32, No. 1, July 1979, pp. 101-136.
} 
${ }^{34}$ Liu, X.-D., Osher, S., and Chan, T., "Weighted Essentially Non-oscillatory Schemes," J. Comput. Phys., Vol. 115, No. 1, Nov. 1994, pp. 200-212.

${ }^{35}$ Johnsen, E., "On the treatment of contact discontinuities using WENO schemes," J. Comput. Phys., Vol. 230, 2011, pp. $8665-8668$

${ }^{36}$ Collins, B. D. and Jacobs, J. W., "PLIF flow visualization and measurements of the RichtmyerMeshkov instability of an air/SF6 interface," J. Fluid Mech., Vol. 464, Aug. 2002, pp. 113-136.

${ }^{37}$ Latini, M., Schilling, O., and Don, W. S., "Effects of WENO flux reconstruction order and spatial resolution on reshocked two-dimensional RichtmyerMeshkov instability," Journal of Computational Physics, Vol. 221, No. 2, Feb. 2007, pp. 805-836. 